\title{
Metachronous Synovial Sarcoma After Treatment of Mixed Germ Cell Tumor in a Child with Complete Gonadal Dysgenesis
} \author{
(D) İclal Gürses ${ }^{5}$, (D) Yüksel Balcı ${ }^{6}$ \\ ${ }^{1}$ Mersin University Faculty of Medicine, Department of Pediatric Oncology, Mersin, Turkey \\ ${ }^{2}$ Mersin University Faculty of Medicine, Department of Medical Oncology, Mersin, Turkey \\ ${ }^{3}$ Mersin University Faculty of Medicine, Department of Pediatrics, Mersin, Turkey \\ ${ }^{4}$ Mersin University Faculty of Medicine, Department of Radiation Oncology, Mersin, Turkey \\ ${ }^{5}$ Mersin University Faculty of Medicine, Department of Pathology, Mersin, Turkey \\ ${ }^{6}$ Mersin University Faculty of Medicine, Department of Radiology, Mersin, Turkey
}

(D) Feryal Karahan ${ }^{1}$, (D) Elvan Çağlar Çıtak ${ }^{1}$, (D) Emel Yaman², (D) Mehmet Alakaya ${ }^{3}$, (D) Fatih Sağcan ${ }^{3}$, (D) Eda Bengi Yılmaz ${ }^{4}$, (D) Funda Kuş ${ }^{5}$,

What is already known on this topic?

Complete 46,XY gonadal dysgenesis (GD) patients show a high predisposition to germ cell tumors. Gonadoblastomas and dysgerminomas are the most frequent histotypes.

\section{What this study adds?}

This is the first report of synovial sarcoma in patients with GD.

\section{Abstract}

Patients with complete XY gonadal dysgenesis (GD) show a high predisposition to germ cell tumors (GCT). Patients with coexistence of GCT and GD have been reported previously. Here we present a 15-year-old girl with mixed GCT and GD who also developed an intraabdominal synovial sarcoma one year after the treatment. This is the first report, to our knowledge, of synovial sarcoma associated with XY GD.

Keywords: Gonadal dysgenesis, synovial sarcoma, dysgerminoma, gonadoblastoma, embryonal carcinoma

\section{Introduction}

A complete 46,XY gonadal dysgenesis (GD) syndrome, known as Swyer syndrome, is characterized by a female phenotype with bilateral streak gonads, normal, female, external genitalia, presence of Müllerian duct and deficient secondary sexual characteristic development with primary amenorrhea (1). Patients with Swyer syndrome show a high predisposition to ovarian cancer. The most frequent observed histotypes are gonadoblastomas and dysgerminomas, followed by Brenner tumors, malignant teratomas and mixed endodermal sinus tumors (2). The lifetime risk of gonadal tumors is in the range of
$15-35 \%$ in patients with GD $(3,4)$. Germ cell tumors (GCT) were reported in patients with GD but not in conjunction with other types of malignancy. In this report we present a 15 years-old girl patient with mixed GCT and GD who underwent a metachronous somatic malignant transformation (SMT) resulting in intraabdominal synovial sarcoma one year after the treatment.

\section{Case Report}

A 15-year old girl was admitted to our hospital with a complaint of abdominal distension. Her abdominal 
examination revealed the presence of a large pelvic mass. Menstrual history revealed that she had never attained menarche. Her height was $171 \mathrm{~cm}[+1.95$ standard deviation score (SDS)], and her weight was $73 \mathrm{~kg}$ (+2.98 SDS). Her body mass index was $25 \mathrm{~kg} / \mathrm{m}^{2}$. Systemic examination showed normal female external genitalia, a "rough" voice, small breasts and a hypoplastic vagina.

Initial hormonal assays showed elevated levels of serum follicle stimulating hormone at $54.3 \mathrm{IU} / \mathrm{mL}$ (3.5-12.5) and luteinizing hormone at $50.82 \mathrm{IU} / \mathrm{mL}$ (2.4-12.6). Other endocrinological evaluations were as follows: progesterone $0.706 \mathrm{ng} / \mathrm{mL}$ (0.4-1.4); estradiol $45 \mathrm{pg} / \mathrm{mL}$ (13-71); testosterone $0.26 \mathrm{ng} / \mathrm{mL}$ (8-80); DHEA-SO4 $327 \mu \mathrm{g} / \mathrm{dL}$ (65-368); and androstenodione $2.02 \mathrm{ng} / \mathrm{mL}$ (0.5-4.8). On contrast-enhanced, computed tomography (CT), there was a mass, separate from the uterus, which was $12 \mathrm{~cm}$ in diameter and which filled the rectouterine space. The mass was mostly multicystic, but also contained solid areas and coarse calcifications (Figure 1A). Lateral to this lesion, there was another solid mass with a diameter of 5 $\mathrm{cm}$ and internal calcifications (Figure 1B). In the left paraaortic region, there were two soft tissue lesions adjacent to each other, with dimensions of $10 \mathrm{~cm}$ and $3 \mathrm{~cm}$ (Figure $1 \mathrm{C})$. The attenuation feature of the larger lesion was similar to that of the lesion in the recto-uterine space, while the smaller appeared to be solid. By radiological appearance, the masses were evaluated as a bilateral malignant ovarian tumor with lymphatic metastases. Laboratory

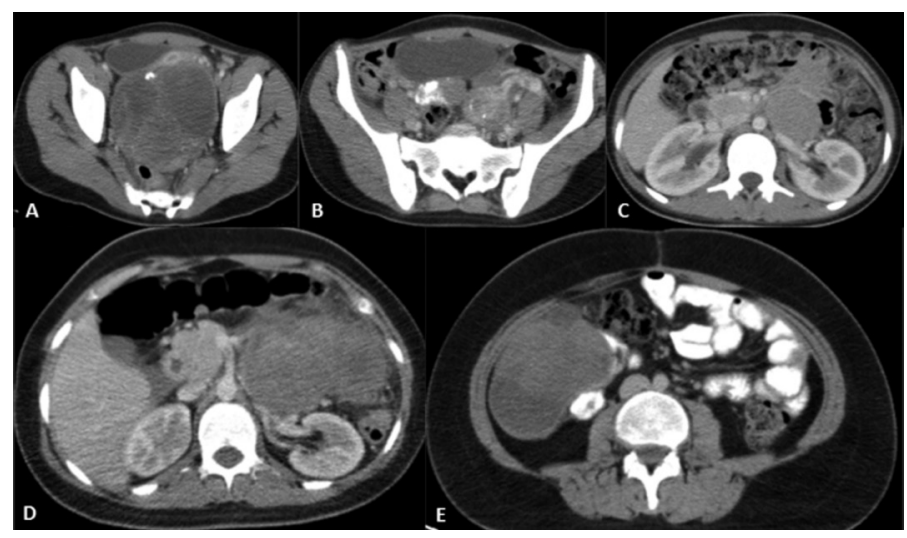

Figure 1. A huge mass with internal cystic components and calcifications, within the cul de sac. Note that the bladder and uterus are displaced anteriorly and the rectum is displaced posteriorly by the lesion (A). Another lesion with calcifications. A small cyst is also visible at the upper level (B), retroperitoneal lymphadenopathy with mild compression on the left renal vein (C). Axial computed tomography image at the renal sinus level shows a huge mass with irregular borders and heterogeneous enhancement within the left retroperitoneal localization, anterior to the left kidney (D). A hypo dense mass with mild irregular contours in the right abdomen at the posterolateral aspect of the caecum and ascending colon (E) tests revealed high levels of serum $\alpha$-fetoprotein (AFP) (19931 IU/mL) and Cancer Antigen 125 (Ca 125) (566.3 $\mathrm{U} / \mathrm{mL}$ ) with normal lactic acid dehydrogenase and beta human chorionic gonadotropin ( $\beta$-hCG) levels. Cytogenetic studies revealed a $46 \mathrm{XY}$ genotype. No germ line deletion or translocation of the sex-determining region $\mathrm{Y}(S R Y)$ gene was detected by fluorescence in situ hybridization. Bilateral gonadectomy, Müllerian duct extraction and tumor resection were performed. Pathological investigation showed dysgerminoma (90\%), embryonal carcinoma (7\%) and gonadoblastoma (3\%) on the left side, a dysgenetic gonad and pure gonadoblastoma on the right side (Figure 1D,E,F). Adjuvant chemotherapy was performed with six cycles of cisplatin/etoposide/bleomycin. The patient was in remission after chemotherapy.

Eighteen months later she was admitted to the emergency department with abdominal pain and distension. A left abdominal mass was detected on physical examination. Abdomino-pelvic CT showed a huge mass with irregular borders and heterogeneous enhancement within the left retroperitoneal region, anterior to the left kidney (Figure 2A). Serum AFP, Ca125, and $\beta$-hCG levels were within the respective normal ranges. The mass was near totally resected. Pathological investigation showed a monophasic synovial sarcoma (Figure 2B). Ifosfamide, mesna and doxorubicin chemotherapy protocol was given for six cycles and radiotherapy was added at a dose of 45 Gy. Twelve

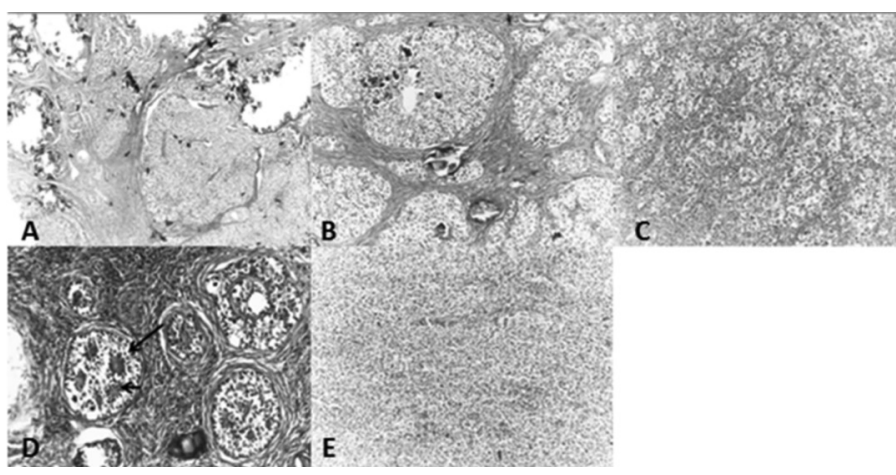

Figure 2. The growth of the gonadoblastoma occurred as rounded nests separated by fibrous stroma that contained significant calcification (hematoxylen and eosin, x40) (A). The germ cells, similar to dysgerminoma cells, in the gonadoblastoma (hematoxylen and eosin, x200) (B). Dysgerminoma. Nests of dysgerminoma cells were separated by fibrous septa containing lymphocytes and plasma cells. The tumor cells had round vesicular nuclei with prominent nucleoli and abundant pale cytoplasm (hematoxylen and eosin, x400) (C). Gonadoblastoma was detected within the streak ovary. The tumor contained a nest of predominantly sex cord-like cells distributed around hyalinized acini (arrow) (hematoxylen and eosin, x400) (D). Synovial sarcoma (monophasic component). Sheets of uniform small spindle cells with ovoid nuclei and scanty cytoplasm (hematoxylen and eosin, x200) (E) 
months later the patient had a further relapse on the right side of the abdomen. Abdominal CT showed a hypodense mass, with mild irregular contours at the posterolateral area of the caecum and ascending colon (Figure 2C). The tumor was partially resected. Ifosfamide/Carboplatin/Etoposide chemotherapy was initiated. Forty-five months after initial diagnosis and 27 months after diagnosis of the synovial sarcoma, the patient died due to resistant/progressive disease.

\section{Discussion}

Patients with GD are phenotypically female with unambiguously female genital appearance at birth and with normal Müllerian structures. The condition typically presents at an age when puberty would be normally expected with primary amenorrhoea and delayed puberty. Although the etiology is not completely understood, $46, \mathrm{XY}$ GD results from failure of testicular development due to disruption of the underlying genetic pathways and several genes, including $S R Y$ (gene deletion or loss of function mutations; Yp11.3), NR5A1 (9q33) and DHH (homozygous or compound heterozygous mutations; 12q13.1) have been implicated. In addition, patients with partial duplications of Xp (including the NROB1 gene) and chromosome $9 \mathrm{p}$ deletions (involving the DMRT1 and DMRT2 genes) may also present with isolated 46,XY complete gonadal dysgenesis (CGD). Rarely mutations in the CBX2 gene and also dublication of DAX1 gene have been considered responsible for the development of 46 , XY CGD $(5,6,7)$. Mutations in the MAP3K1 gene (located on chromosome $5 q$ ) that cause downstream alterations in the MAP kinase signaling pathway have been identified (8).

Patients with GD have a $30 \%$ risk of development of gonadoblastoma with a 50-60\% risk of malignant transformation, typically to dysgerminoma (9). The risk of malignancy in patients with GD increases with age; it has been reported that the risk is $50-70 \%$ in the third decade and as high as $80 \%$ in the fourth (10). In patients with primary amenorrhoea, GD should always be kept in mind due to the high risk of malignant transformation. Bilateral gonadectomy is advised as soon as the diagnosis is made (11). Unfortunately, our patient had bilateral gonadectomy and Müllerian duct extractions only after the disease had progressed to bilateral GCT.

Gonadoblastomas are rare, mixed, germ cell, sex cord, stromal tumors, almost exclusively seen in patients with underlying gonadal disorders. Germ cells in dysgenetic gonads are genetically unstable and tumorigenic because patients with GD have a higher risk of development of germ cell tumor. It has been hypothesized that the Y chromosome contains a gonadoblastoma locus, responsible for this benign tumor, that may also develop bilaterally and coexist with other neoplasms, such as dysgerminoma (12). Radaković et al (13) reported that $55 \%$ of patients suffering from GD were diagnosed with gonadoblastoma or dysgerminoma. Cases of co-occurrence of gonadoblastoma with dysgerminoma and gonadoblastoma with choriocarcinoma have also been reported (12). It is assumed that gonadoblastomas are unstable and may result in choriocarcinoma (14). Our patient had a pure gonadoblastoma in her right ovary and dysgerminoma, embryonal carcinoma and gonadoblastoma on the left side.

GCTs include a diverse group of tumors that arise from primordial germ cells, either in the gonads or in nongonadal sites. SMT of a GCT means the occurrence of somatic non-germ cell malignancy. Faure Conter et al (15) reported that SMT associated with GCT in children is rare and that these are aggressive tumors with various primary lesions, various GCT histologic subtypes, and poor overall prognosis. Although the presentation is mostly synchronous in children, most of the adult cases are metachronous. Giannatempo et al (16) reported that the median delay between SMT and GCT was four years with a maximum of 18 years. They also reported that SMT occurred concurrently with GCT in only $40 \%$ of patients. Different studies showed that $78.5-100 \%$ of SMT were diagnosed at the same time as the GCT diagnosis. Different malignant tumors such as rhabdomyosarcoma, peripheral primitive neuroectodermal tumors, adenocarcinoma, squamous cell carcinoma, osteosarcoma, angiosarcoma, leukemia, neurosarcoma, undifferentiated sarcoma, myxoid sarcoma, fusiform cell sarcoma, bronchoalveolar sarcoma and thyroid carcinoma have been reported to arise in GCT (17). Our patient had a metachronous presentation, similar to that reported in adult cases, after 18 months from the initial diagnosis and she had no GCT at that time. Tumor markers were negative at the diagnosis of synovial sarcoma.

In most of the reported cases with SMT, primary diagnoses were teratoma $(15,16,18)$. GCTs have a capacity to display totipotential differentiation. Some authors explain this condition with malignant transformation of the yolk sac or with teratoma cells while others propose a divergent differentiation of primordial stem cells toward GCT and SMT $(15,16,18,19)$. Specific chromosomal changes trigger this transformation. The presence of isochromosome $12 \mathrm{p}, \mathrm{a}$ specific marker of GCT and of chromosomal abnormalities in MT of non-GCT, strongly favor this hypothesis. We did not investigate the presence of isochromosome $12 p$ in our patient. It is possible that this chromosomal aberration may have caused the metachronous tumor development. The patient received cisplatin/etoposide/bleomycin for the treatment of GCT. The most well known late complications of etoposide are dose related myelodysplastic syndrome and secondary acute myeloid leukemia $(20,21,22)$. A similar complication (leukemia) is reported for cisplatine in many 
studies $(23,24)$. The most important late complication of bleomycin is pulmonary toxicity. These complications, especially the secondary cancers usually occur five years after treatment. In our patient, synovial sarcoma occurred 18 months after the treatment and we believe that the tumor developed metachronously, rather than as a complication of the chemotherapeutics given in this case.

We could find no other case reports of patients with GD developing synovial sarcoma after GCT. Thus, to the best of our knowledge, this is the first report of GD with synovial sarcoma following GCT.

Bilateral gonadectomy and Müllerian duct extractions have to be considered for newly diagnosed patients with GD as a risk reducing strategy for development of malignancy. Patients with GCT and with chromosomal or genetic defects must be carefully followed and observed because of the high risk for development of synchronous or metachronous SMT.

\section{Ethics}

Informed Consent: Written inform consent for publication of the data was given by the patient's parents.

Peer-review: Externally peer-reviewed.

\section{Authorship Contributions}

Concept: Elvan Çağlar Çıtak, Emel Yaman, Design: Elvan Çağlar Çıtak, Feryal Karahan, Mehmet Alakaya, Data Collection and Processing: Eda Bengi Yılmaz, Funda Kuş, İclal Gürses, Yüksel Balc1, Analysis and Interpretation: Elvan Çağlar Çıtak, Emel Yaman, Fatih Sağcan, Eda Bengi Yılmaz, Feryal Karahan, Mehmet Alakaya, Yüksel Balcı, Literature Research: Fatih Sağcan, Feryal Karahan, Mehmet Alakaya, Writing: Elvan Çağlar Çıtak, Eda Bengi Yilmaz.

Financial Disclosure: The authors declared that this study received no financial support.

\section{References}

1. Michala L, Goswami D, Creighton SM, Conway GS. Swyer syndrome: presentation and outcomes. BJOG 2008;115:737-741.

2. Zhu HL, Bao DM, Wang Y, Shen DH, Li Y, Cui H. Swyer's syndrome with mixed ovarian malignant germ cell tumor and ovarian gonadoblatoma. Chin Med J 2016;129:1752-1754.

3. Kulathilake DT, Jayasundara C. A germ cell tumor in a patient with Swyer syndrome with ambiguous genitalia. BMC Res Notes 2015;8:747.

4. Hoepffner W, Horn LC, Simon E, Sauerbrei G, Schröder H, ThammMücke B, Bennek J, Kiess W. Gonadoblastomas in 5 patients with 46,XY gonadal dysgenesis. Exp Clin Endocrinol Diabetes 2005;113:231-235.

5. King TF, Conway GS. Swyer Syndrome. Curr Opin Endocrinol Diabetes Obes 2014;21:504-510.

6. Du X, Zhang X, Li Y, Han Y. 46 XY female sex reversal syndrome with bilateral gonadoblastoma dysgerminoma. Exp Ther Med 2014;8:1102-1104.
7. Sukumaran A, Desmangles JC, Gartner LA and Buchlis J. Duplication of dosage sensitive sex reversal area in a 46, XY patient with normal sex determining region of $Y$ causing complete sex reversal. J Pediatr Endocrinol Metab 2013;26:775-779.

8. Granados A, Alaniz VI, Mohnach L, Barseghyan H, Vilain E, Ostrer H, Quint EH, Chen M, Keegan CE. MAP3K1-related gonadal dysgenesis: Six new cases and review of the literature. Am J Med Genet C Semin Med Genet 2017;175:253-259

9. Massanyi EZ, DiCarlo HN, Migeon CJ, Gearhart JP. Review and management of 46, XY disorders of sex development. J Pediatr Urol 2013;9:368-379.

10. Uehara S, Funato T, Yaegashi N, Suziki H, Sato J, Sasaki T, Yajima A. SRY mutation and tumor formation on the gonads of XY pure gonadal dysgenesis patients. Cancer Genet Cytogenet 1999;113:78-84.

11. Keskin M, Savas-Erdeve S, Kurnaz E, Cetinkaya S, Karaman A, Apaydin $\mathrm{S}$, Aycan Z. Gonadoblastoma in a patient with $46, \mathrm{XY}$ complete gonadal dysgenesis. Turkish J Pediatr 2016;58:538-540.

12. Zielinska D, Zajaczek S, Rzepka-Gorska I. Tumors of dysgenetic gonads in Swyer syndrome. J Pediatr Surg 2007;42:1721-1724.

13. Radaković B, Jukić S, Buković D, Ljubojević N, Cima I. Morphology of gonads in pure XY gonadal dysgenesis. Coll Antropol 1999;23:203-211.

14. Beaulieu BM, Bouron-Dal SD, Maietta A, Fournet JC, Blumenkrantz $\mathrm{M}$, Brochu P, Lemieux N. Coexistence of a choriocarcinoma and a gonadoblastoma in the gonad of a 46, XY female: A single nucleotide polymorphism array analysis. Pediatr Dev Pathol 2010;13:66-71.

15. Faure Conter C, Fresneau B, Thebaud E, Bertrand A, Dijoud F, Rome A, Dumesnil C, Castex MP, Ghanem A, Orbach D. Two Tumors in 1: What should be the therapeutic target? Pediatric germ cell tumor with somatic malignant transformation. J Pediatr Hematol Oncol 2017;39:388-394.

16. Giannatempo P, Pond GR, Sonpavde G, Albany C, Loriot Y, Sweeney CJ, Salvioni R, Colecchia M, Nicolai N, Raggi D, Rice KR, Flack CK, El Mouallem NR, Feldman H, Fizazi K, Einhorn LH, Foster RS, Necchi A, Cary C. Treatment and clinical outcomes of patients with teratoma with somatic-type malignant transformation: an International collaboration. J Urol 2016;196:95-100.

17. Unal E, Koksal Y, Toy H, Gunel E, Acikgozoglu S. Neuroblastoma arising from unresected sacrococcygeal teratoma in a child. J Pediatr Hematol Oncol 2010;32:233-235.

18. Cabral FC, Krajewski KM, Rosenthal MH, Hirsch MS, Howard SA. Teratoma with malignant transformation: report of three cases and review of the literature. Clin Imaging 2014;38:589-593.

19. Ulbright TM. Germ cell tumors of the gonads: a selective review emphasizing problems in differential diagnosis, new appreciated, and controversial issues. Mod Pathol 2005;18(Suppl 2):61-79.

20. Ezoe S. Secondary leukemia associated with the anti-cancer agent, etoposide, a topoisomerase II inhibitor. Int J Environ Res Public Health 2012;9:2444-2453.

21. Adamson PC, Balis FM, Berg S, Blaney SM. General Principles of Chemotherapy. In: Pizzo PA, Poplack DG (eds). Principles and Practice of Pediatric Oncology. Lippincott Williams \& Wilkins, 2006;290-365.

22. Bhatia S, Blatt J, Meadows A. Late Effects of Childhood Cancer and Its Treatment. In: Pizzo PA, Poplack DG (eds). Principles and Practice of Pediatric Oncology. Lippincott Williams \& Wilkins, 2006;1490-1514.

23. Philpott NJ, Elebute MO, Powles R, Treleaven JG, Gore M, Dainton MG, Min T, Swansbury GJ, Catovsky D. Platinium agents and secondary myeloid leukemia: two cases related only with platinium-based drug. Br J Haematol 1996;93:884-887.

24. Travis LB, Andersson M, Gospodarowicz M, van Leeuwen FE, Bergfeldt K, Lynch CF, Curtis RE, Kohler BA, Wilklund T, Storm H, Holowaty E, Hall P, Pukkala E, Sleijfer DT, Clarke EA, Boice JD Jr, Stovall M, Gilbert E. Treatment-associated leukemia following testicular cancer. J Natl Cancer Inst 2000;92:1165-1171. 